adding back the purified factors to cellfree systems prepared from cells subjected to such physiological stresses ${ }^{8,9}$.

Furthermore, modulation of intracellular $p \mathrm{H}$ and ionic conditions (especially $\left.\mathrm{Ca}^{2+}\right)$, such as occurs when quiescent cells are stimulated by growth factors or serum, is capable of regulating translation in mammalian cells (E.C. Henshaw and R. Panniers, University of Rochester Cancer Center). There are therefore many aspects in common between translational control in eggs and the responses of somatic cells to growthregulatory conditions.
1. Raff, R.A. in Cell Biology, A Comprehensive Treatise (eds Prescott, C.D.M. \& Goldstein, L.) 107-136 (Academic, Orlando, 1980).

2. Colin, A.M. et al. Devl Biol. 123, 354-363 (1987).

3. Pain, V.M. Biochem.J. 235, 625-637 (1986)

4. Pestka, S., Langer, J.A., Zoon, K.C. \& Samuel, C.E. A. Rev. Biochem. 56, 727-777 (1987).

Huang, W-I., Hansen, L.J., Merrick, W.C. \& Jagus, R. Proc.natn.Acad.Sci.U.S.A. 84, 6359-6363 (1987).

6. Audet, R.G., Goodchild, J. \& Richter, J.D. Devl Biol. 11, 58-68 (1987)

7. Duncan, R., Milburn, S.C. \& Hershey, J.W.B. J.biol.Chem 262, 380-388 (1987)

8. Panniers, R., Stewart, E.B., Merrick, W.C. \& Henshaw, E.C. J.biol.Chem. 260, 9648-9653 (1985)

9. Clemens, M.J. et al. J.biol.Chem. 262, 767-771 (1987).

Mike Clemens is in the Department of Biochemistry, St George's Hospital Medical School, London SWI7ORE, UK.

\title{
Cosmology
}

\section{Can nucleons close the Universe?}

\section{K. A. Olive}

THE standard Big Bang model of the early Universe is tested observationally at two very different epochs. First, the observation of the 3-K microwave background discovered by Penzias and Wilson ${ }^{1}$ probes back to the time when the age of the Universe was about $10^{5}$ yr (today the age of the Universe is about $10^{10} \mathrm{yr}$ ). Second, Big Bang nucleosynthesis (see, for example, ref. 2), the era when the light nuclei $\mathrm{D},{ }^{3} \mathrm{He},{ }^{4} \mathrm{He}$ and ${ }^{7} \mathrm{Li}$ were produced, occurred about 1 min after the Big Bang. The microwave background radiation confirms the existence of our hot past and provides invaluable information regarding the overall isotropy of the Universe. Because nucleosynthesis probes back to much hotter temperatures (about $10^{10} \mathrm{~K}$ ) and hence much earlier times, however, the abundances of the light nuclei are sensitive to many microphysical processes predicted in theories of high-energy physics. The effects of the transition from quarks and gluons (the constituents of nuclear matter) to hadrons (normal nuclear matter) before nucleosynthesis have now been investigated ${ }^{3-6}$ and contrasted with standard-model predictions.

The fact that the standard model of nucleosynthesis provides excellent agreement with the observational determination of the light-nuclear abundances allows the theories of particle physics to be constrained. A prime example of a nucleosynthesis constraint is the restriction on the number of light neutrino types $^{7.8}$. One additional neutrino type beyond the known three begins to produce an overabundance of ${ }^{4} \mathrm{He}$. Any more beyond that would cause a conflict between theory and observations.

Agreement for all of the numerical abundances, which span ten orders of magnitude $\left(\mathrm{D} / \mathrm{H} \simeq 10^{-5},{ }^{3} \mathrm{He} / \mathrm{H} \simeq 10^{-5}\right.$, ${ }^{4} \mathrm{He} / \mathrm{H} \simeq 0.08$ and ${ }^{7} \mathrm{Li} / \mathrm{H} \simeq 10^{-10}$ ), occurs only for a limited range of the ratio $\eta$ of the number of baryons (protons and neutrons) to the number of photons, $3 \times 10^{-10}<\eta<6 \times 10^{-10}$. In addition, because the density of photons, $n_{\gamma}$ is known (by measuring the temperature of the microwave background), we can relate $\eta$ to the ratio $\Omega_{\mathrm{B}}$ of the total mass density in baryons, $\varrho_{\mathrm{B}}$, to the critical density, $\varrho_{c}$, needed to close the Universe: $\Omega_{\mathrm{B}}=\varrho_{\mathrm{B}} / \varrho_{\mathrm{c}}=3.6 \times 10^{7} \eta h_{0}^{-2}\left(T_{0} / 2.7 \mathrm{~K}\right)^{3}$, where $H_{0}=100 h_{0} \mathrm{~km} \mathrm{~s}^{-1} \mathrm{Mpc}^{-1}$ is the present value of the Hubble parameter and $T_{0}$ is the present temperature of the microwave background. The maximum value of $\Omega_{\mathrm{B}}$, obtained with $\eta=6 \times 10^{-10}$, $h_{0}=0.4$ and $T_{0}=2.8 \mathrm{~K}$, is 0.15 , thus excluding the possibility that the Universe is closed (so that its expansion asymptotically stops) by ordinary matter.

Recently there have been some interesting proposals for altering the standard scheme for nucleosynthesis ${ }^{3-6}$. These use the effects of a phase transition between normal, hadronic matter and quark-gluon matter which is thought to have existed about $10^{-5} \mathrm{~s}$ after the Big Bang. In particular, depending on the largely unknown details of this transition, baryon density fluctuations can arise as a result of the difficulty in producing a baryon with mass $m_{\mathrm{B}} \simeq 1 \mathrm{GeV}$ at a temperature $k T=200$ $\mathrm{MeV}$ ( $k$ is Boltzmann's constant). If indeed baryon production is suppressed in the hadron phase, an excess will appear in the quark phase which, as the transition proceeds, occupies less and less space, thus resulting in a baryon density fluctuation.

It has been known for some time that baryon density fluctuations could affect Big Bang nucleosynthesis, but the effects were rather minimal. Applegate and Hogan ${ }^{3}$ suggested, and now with Scherrer ${ }^{4}$ show, that in this primordial medium, the mean free paths of neutrons and protons are different, leading to a segregation of neutrons and protons. The Universe after the transition is then divided into neutron-rich and neutron- poor regions. The effect of this inhomogeneity on nucleosynthesis can be great.

Because the abundances of $\mathrm{D},{ }^{3} \mathrm{He}$ and ${ }^{4} \mathrm{He}$ as a function of $\eta$ are largely monotonic, a suitable choice of transition parameters can hold the average abundance of each of these nuclei constant, as $\eta$ is increased to the point where $\Omega_{\mathrm{B}}=1$. But ${ }^{7} \mathrm{Li}$ abundance does not change monotonically as a function of $\eta$. In the standard model of nucleosynthesis, there is a minimum for the abundance of ${ }^{7} \mathrm{Li}$ at $\eta=$ $3 \times 10^{-10}$ for which the fraction of the mass of the Universe in ${ }^{7} \mathrm{Li}, X\left({ }^{7} \mathrm{Li}\right)=4 \times 10^{-10}$. Either increasing or decreasing $\eta$ results in a higher ${ }^{7} \mathrm{Li}$ abundance ${ }^{8}$. Thus, although Alcock et al. ${ }^{6}$ can replicate standard Big Bang nucleosynthesis results for $\mathrm{D},{ }^{3} \mathrm{He}$ and ${ }^{4} \mathrm{He}$ and $\Omega_{\mathrm{B}}=1$, they find that the abundance of ${ }^{7} \mathrm{Li}$ ranges from $3 \times 10^{-8}$ to $10^{-7}$, which should be compared with observational determinations of ${ }^{7} \mathrm{Li}$ abundance.

Because ${ }^{7} \mathrm{Li}$ can be both produced and destroyed in other astrophysical sites such as stars and cosmic-ray spallation, it is not a trivial task to extract the primordial ${ }^{7} \mathrm{Li}$ abundance from the observations. Indeed, observations of ${ }^{7} \mathrm{Li}$ in population II stars imply $X\left({ }^{7} \mathrm{Li}\right) \simeq 6 \times 10^{-10}$ and in population I stars $X\left({ }^{7} \mathrm{Li}\right) \simeq 7 \times 10^{-9}$, although there are arguments ${ }^{9}$ that suggest that population II abundances more accurately represent the primordial abundances. In any case, both measures of ${ }^{7} \mathrm{Li}$ abundance fall well short of the values produced in nucleosynthesis calculations based on inhomogeneities induced by the quark-hadron transition.

I would like to stress that this should not be construed as a negative result. Instead, these results can be used to place constraints on the quark-hadron transition as has been done by Reeves ${ }^{10}$. Using both measures of $X\left({ }^{7} \mathrm{Li}\right)$, he places limits on the unknown parameters of the quarkhadron transition, such as the baryonnumber contrast and the fractional mass in the two phases. These constraints imply that $\eta$ lies in the restricted range $1 \times 10^{-10}-6 \times 10^{-10}$, or $\Omega_{\mathrm{B}} \leqslant 0.15$ as before, and thus exclude the possibility of closing the Universe with nucleons.

1. Penzias, A.A. \& Wilson, R.W. Astrophys. J. 142, 419 (1965).

2. Bosegard, A. \& Steigman, G. A. Rev. astr. Astrophys. 23, 319-378 (1985).

3. Applegate, J.H. \& Hogan, C.J. Phys. Rev. D31, $3037-$ 3045 (1985).

4. Applegate, J.H., Hogan. C.J. \& Scherrer, R.J. Phys. Rev. D35, 1151-1160 (1987).

5. Sale, K.E. \& Mathews, C.J. Astrophys, J. 302, L1-LA (1986).

6. Alcock, C., Fuller, G.M. \& Mathews, G.J. Astrophys. J. 320, 439-447 (1987).

7. Steigman, G., Schramm, D.N. \& Gunn, J.E. Phys. Lett. 66B, 202-204 (1977).

8. Yang, J., Turner, M.S., Steigman, G., Schramm, D.N. \& Olive, K.A. Astrophys. J. 281, 493-511 (1984)

9. Kawano, L., Schramm, D.N. \& Steigman, G. Astrophys. $J$. (in the press).

10. Reeves, H. Proc. Int. Sch. Phys. Enrico Fermi (in the press).

K.A. Olive is in the School of Physics and Astronomy, University of Minnesota, Minneapolis, Minnesota 55455, USA. 